\title{
Event-Based Control for Average Consensus of Wireless Sensor Networks with Stochastic Communication Noises
}

\author{
Chuan Ji, Wuneng Zhou, and Huashan Liu \\ College of Information Sciences and Technology, Donghua University, Shanghai 200051, China \\ Correspondence should be addressed to Wuneng Zhou; zhouwuneng@163.com
}

Received 1 November 2013; Accepted 20 November 2013

Academic Editor: Guoliang Wei

Copyright (C) 2013 Chuan Ji et al. This is an open access article distributed under the Creative Commons Attribution License, which permits unrestricted use, distribution, and reproduction in any medium, provided the original work is properly cited.

This paper focuses on the average consensus problem for the wireless sensor networks (WSNs) with fixed and Markovian switching, undirected and connected network topologies in the noise environment. Event-based protocol is applied to each sensor node to reach the consensus. An event triggering strategy is designed based on a Lyapunov function. Under the event trigger condition, some sufficient conditions for average consensus in mean square are obtained. Finally, some numerical simulations are given to illustrate the effectiveness of the results derived in this paper.

\section{Introduction}

Wireless sensor network (WSN) has attracted significant attention as an emerging communication architecture. It has many practical applications in such areas as robotics, surveillance and environment monitoring, and information collection.

A WSN can be viewed as a multiagent system (MAS) from a network-theoretic perspective. Each node represents a sensor and each edge performs information exchange between sensors. In some cases, the agreement is a common value which may be the average of the initial states of the system, is often called average consensus, and has wide application background in the areas such as formation control [1], distributed filtering [2], and distributed computation [3]. It means to achieve the accordance of the states of MAS. In [4], Olfati-Saber and Murray consider the average consensus control for the directed and undirected networks with fixed and switching topologies. In [5], Kingston and Beard extend the results of [4] to the discrete-time models and weakened the condition of instantaneous strong connectivity. In [6], Xiao and Boyd consider the distributed averaging consensus of the networks with fixed and undirected topologies. In [7], Q. Zhang and J. Zhang design a distributed consensus protocol to analyze the multiagent systems in uncertain communication environments including the communication noises and Markov topology switches. In [8], Wang et al. investigate the $\mathrm{H} \infty$ consensus control problem for a class of discrete time-varying multiagent systems with both missing measurements and parameter uncertainties. Also, the distributed estimation problems over sensor networks have been widely discussed in [9-11].

For the node of the WSNs with limited energy, control over networks with limited resources is a challenging task. Consequently, the most important problem in WSN is the energy consumption, which is directly proportional to the transmit power of the information exchange between the sensors. The event-based control can facilitate the efficient usage of the resources. Based on the event-based control mechanism, it can reduce the useless communication between neighboring agents along with the energy consumption. In [12], Wang and Lemmon discuss the event-triggered data transmission in distributed networked control systems with packet loss and transmission delays. In [13], Mazo and Tabuada focus on reducing the number of messages from sensors to controllers and from controllers to actuators by the use of the decentralized event-triggered mechanism. In [14], Seyboth et al. propose a novel control strategy for multiagent coordination with event-based broadcasting. The proposed control strategy guarantees either asymptotic convergence to 
average consensus or convergence to a ball centered at the average consensus. In [15], Meng and Chen study an average consensus problem for multiagent systems by event-based control, which is used on each agent to drive the state to their initial average eventually.

The main contribution of this paper is studying average consensus problem for the WSN with fixed topology and Markovian switching topology in the noise environment; the event-based average consensus control protocol is designed for the WSN based on a Lyapunov function. Under the event trigger condition, sufficient conditions for average consensus in mean square are obtained.

The remainder of the paper is organized as follows. In Section 2, some concepts in graph theory are described, and the problem to be investigated is formulated. In Section 3, the main results are presented. In Section 4, some numerical examples show the reliability of the main results. In Section 5, some conclusions are given.

\section{Problem Formulation and Preliminaries}

2.1. Concepts in Graph Theory. Let $\mathscr{G}=\{\mathscr{V}, \mathscr{E}, \mathscr{A}\}$ be an undirected graph, where $\mathscr{V}=\{1,2, \ldots, n\}$ is the set of nodes, node $i$ represents the $i$ th sensor node, $\mathscr{E}$ is the set of edges, and an edge in $\mathscr{G}$ is denoted by an ordered pair $(j, i) .(j, i) \in \mathscr{E}$ if and only if the $j$ th sensor node can send information to the $i$ th sensor node directly. The neighborhood of the $i$ th sensor node is denoted by $N_{i}=\{j \in \mathscr{V} \mid(j, i) \in \mathscr{E}\}$.

$\mathscr{A}=\left[a_{i j}\right] \in \mathbb{R}^{n \times n}$ is called the adjacency matrix of $\mathscr{G}$. For any $i, j \in \mathscr{V}, a_{i j} \geq 0$, and $a_{i j}>0 \Leftrightarrow j \in N_{i}$. $\operatorname{deg}_{\text {in }}(i)=$ $\sum_{j=1}^{n} a_{i j}$ is called the in-degree of $i$; $\operatorname{deg}_{\text {out }}(i)=\sum_{j=1}^{n} a_{i j}$ is called the out-degree of $i ; L=\mathscr{D}-\mathscr{A}$ is called the Laplacian matrix of $\mathscr{G}$, where $\mathscr{D}=\operatorname{diag}\left(\operatorname{deg}_{\text {in }}(1), \ldots, \operatorname{deg}_{\text {in }}(n)\right)$. Its eigenvalues are real and can be ordered as

$$
\lambda_{1} \leq \lambda_{2} \leq \cdots \leq \lambda_{n}
$$

with $\lambda_{1}=0$ and $\lambda_{2}$ being the smallest nonzero eigenvalue for connected graphs.

2.2. Average Consensus for WSNs. In this paper, we study the average consensus control for a WSN with dynamics

$$
\dot{x}_{i}(t)=u_{i}(t),
$$

where $x_{i}(t) \in \mathbb{R}$ is the state of the $i$ th sensor and $u_{i}(t) \in \mathbb{R}$ is the control input. The initial state $x_{i}(0)$ is deterministic.

Each sensor includes a digital microprocessor and dynamics. The microprocessor of sensor $i$ monitors its own measurement value $x_{i}(t)$ continuously and decides when to communicate with the neighboring sensors by broadcasting the actual measurement value. Therefore the latest broadcasted value of sensor $i$ can be described by the piecewise constant function

$$
\widehat{x}_{i}(t)=x_{i}\left(t_{k}^{i}\right), \quad t_{k}^{i} \leq t<t_{k+1}^{i},
$$

where $t_{0}^{i}, t_{1}^{i}, \ldots$ is sequence of event-times of sensor $i$. We can see that the discrete-time signal $x_{i}\left(t_{k}^{i}\right)$ is converted into the continuous-time signal $\widehat{x}_{i}(t)$.
The $i$ th sensor can receive information from its neighbors

$$
\widehat{y}_{j i}(t)=\widehat{x}_{j}(t)+\sigma_{j i} \omega_{j i}(t),
$$

where $y_{j i}(t)$ denotes the measurement of the $j$ th sensor's state $x_{j}(t)$ by the $i$ th sensors, $\left\{\omega_{j i}(t) \mid i, j=1,2, \ldots, n\right\}$ are the communication noises, and $\sigma_{j i}$ is the noise intensity function.

For the dynamic network $(\mathscr{G}, X)$, we give the event-based consensus protocl.

$$
u_{i}(t)=\sum_{j \in N_{i}} a_{i j}\left(\widehat{y}_{j i}(t)-\widehat{x}_{i}(t)\right) .
$$

The dynamics of agent $i$ for $t \in\left[t_{k}^{i}+l h, t_{k}^{i}+l h+h\right)$ is given by

$$
\begin{aligned}
\dot{x}_{i}(t)= & \sum_{j \in N_{i}(t)} a_{i j}\left[x_{j}\left(t_{k^{\prime}}^{j}\right)-x_{i}\left(t_{k}^{i}\right)+\sigma_{j i} \omega_{j i}(t)\right] \\
= & \sum_{j \in N_{i}(t)} a_{i j}\left[x_{j}\left(t_{k}^{i}+l h\right)-x_{i}\left(t_{k}^{i}+l h\right)\right] \\
& +\sum_{j \in N_{i}(t)} a_{i j}\left[x_{i}\left(t_{k}^{i}+l h\right)-x_{i}\left(t_{k}^{i}\right)\right] \\
& +\sum_{j \in N_{i}(t)} a_{i j}\left[x_{j}\left(t_{k^{\prime}}^{j}\right)-x_{j}\left(t_{k}^{i}+l h\right)\right] \\
& +\sum_{j \in N_{i}(t)} a_{i j} \sigma_{j i} \omega_{j i}(t) \\
= & \sum_{j \in N_{i}(t)} a_{i j}\left[x_{j}\left(t_{k}^{i}+l h\right)-x_{i}\left(t_{k}^{i}+l h\right)\right] \\
& +\sum_{j \in N_{i}(t)} a_{i j}\left[e_{j}\left(t_{k}^{i}+l h\right)-e_{i}\left(t_{k}^{i}+l h\right)\right] \\
& +\sum_{j \in N_{i}(t)} a_{i j} \sigma_{j i} \omega_{j i}(t),
\end{aligned}
$$

where $t_{k^{\prime}}^{j}$ is defined as

$$
t_{k^{\prime}}^{j}=\max \left\{t \mid t \in\left\{t_{k}^{j}, k=0,1, \ldots\right\}, t \leq t_{k}^{i}+l h\right\} .
$$

Substituting the protocol (5) into the system (2) leads to

$$
d x(t)=-[L(x(k h)+e(k h))] d t+G d W(t),
$$

where $L$ is the Laplacian matrix of $\mathscr{G}, G$ is the noise intensity matrix, and $W(t)=\left(W_{1}(t), \ldots, W_{n}(t)\right)^{T}$ is an $n$-dimensional Brownian motion.

The event condition for agent $i$ has the following form:

$$
\lambda_{n}\left\|x_{i}\left(t_{k}^{i}+l h\right)\right\|^{2} \leq \lambda_{2}\left\|e_{i}\left(t_{k}^{i}+l h\right)\right\|^{2}+2\|G\|^{2},
$$

where

$$
e_{i}\left(t_{k}^{i}+l h\right)=x_{i}\left(t_{k}^{i}\right)-x_{i}\left(t_{k}^{i}+l h\right) .
$$

Remark 1. Each sensor broadcasts its state information to the neighbors and also receives state information from its neighbors for event detection at each sampling instant. The event detector in (9) guarantees that it reduces the sensor energy consumption and network bandwidth usage because it only checks the event condition at discrete sampling instants. 
Next, we consider the average consensus control protocol for the system (8) as follows:

$$
\begin{aligned}
d \delta(t)= & -L[\delta(k h)+(I-J) e(k h)] d t \\
& +(I-J) G d W(t),
\end{aligned}
$$

where $\delta(t)=x(t)-J x(t)$ and $J=(1 / n) 1_{n} 1_{n}^{T}$.

If we define

$$
\begin{gathered}
f(\delta, t)=-L[\delta(k h)+(I-J) e(k h)], \\
g(\delta, t)=(I-J) G,
\end{gathered}
$$

then (11) becomes

$$
d \delta(t)=f(\delta, t) d t+g(\delta, t) d W(t) .
$$

In WSNs, each sensor node communicates with other sensor nodes through the unreliable networks. If the communication channel between sensors $i$ and $j$ is $(j, i) \in \mathscr{E}_{f}$, and $\mathscr{E}_{f}$ is the set of the communication channels which probably lost the signal, then the time-varying topologies under link failure or creation can be described by the Markov switching topology.

Let $\left\{r(t)_{t \geq 0}\right\}$ be a right-continuous Markov chain on the probability space taking values in a finite state set $S=$ $\{1,2, \ldots, N\}$ with generator $\Gamma=\left(\gamma_{i j}\right)_{N \times N}$ given by

$$
P\{r(t+\Delta)=j \mid r(t)=i\}= \begin{cases}\gamma_{i j} \Delta+o(\Delta), & \text { if } i \neq j, \\ 1+\gamma_{i i} \Delta+o(\Delta), & \text { if } i=j,\end{cases}
$$

where $\Delta>0$ and $\gamma_{i j} \geq 0$ is the transition rate from $i$ to $j$ if $i \neq j$, while

$$
\gamma_{i i}=-\sum_{j \neq i} \gamma_{i j}
$$

and $r(0)=r_{0}$.

We denote the undirected communication graph by $\mathscr{G}=$ $\{\mathscr{G}(1), \mathscr{G}(2), \ldots, \mathscr{G}(N)\}$, where $\mathscr{G}(k)=\left\{\mathscr{V}, \mathscr{E}_{\mathscr{G}(k)}, \mathscr{A}_{\mathscr{G}(k)}\right\}$ is the undirected graph. Denote the topology graph by $\mathscr{G}_{t}$ at moment $t(t \geq 0)$, so $r(t)=k$ if and only if $\mathscr{G}_{t}=\mathscr{G}(k)$.

Under Markovian switching topology, we have

$$
\begin{aligned}
d x(t)= & -[L(r(t))(x(k h)+e(k h))] d t \\
& +G(r(t)) d W(t), \\
d \delta(t)= & -L(r(t))[\delta(k h)+(I-J) e(k h)] d t \\
& +(I-J) G(r(t)) d W(t) .
\end{aligned}
$$

So, under this condition, the event condition for agent $i$ has the following form:

$$
\begin{aligned}
\lambda_{n}(L(r(t)))\left\|x_{i}\left(t_{k}^{i}+l h\right)\right\|^{2} \\
\quad \leq \lambda_{2}(L(r(t)))\left\|e_{i}\left(t_{k}^{i}+l h\right)\right\|^{2}+2\|G\|^{2},
\end{aligned}
$$

where

$$
e_{i}\left(t_{k}^{i}+l h\right)=x_{i}\left(t_{k}^{i}\right)-x_{i}\left(t_{k}^{i}+l h\right)
$$

\section{Main Results}

In this section, we consider the average consensus for the WSNs with fixed topology and Markovian switching topology by event-based control.

\subsection{WSNs with the Fixed Topology}

Theorem 2. Consider the system (8) over a connected communication graph driven by event condition in (9). Then the system (11) is asymptotically stable in mean square; it means that the system (8) reaches average consensus in mean square if $0 \leq h \leq 1 / 2 \lambda_{n}$.

Proof. Define the function $V: R^{n} \times R_{+} \rightarrow R^{+}$by

$$
V(\delta, t)=\frac{1}{2} \delta^{T}(t) \delta(t)
$$

Computing $d V(\delta, t)$ along the trajectory generated by the system (11) for any $t \in[k h,(k+1) h)$, we have

$$
\begin{aligned}
d V(\delta, t)=[ & \left.\delta^{T}(t) f(\delta, t)+\operatorname{trace}\left[g^{T}(\delta, t) g(\delta, t)\right]\right] d t \\
& +\delta^{T}(t)(I-J) G d W(t) \\
= & -\delta^{T}(t) L[\delta(k h)+(I-J) e(k h)] \\
& \left.+\|(I-J) G\|^{2}\right] d t+\delta^{T}(t)(I-J) G d W(t) \\
= & (t-k h)[\delta(k h)+(I-J) e(k h)]^{T} \\
& \times L^{T} L[\delta(k h)+(I-J) e(k h)] \\
& -\delta^{T}(k h) L[\delta(k h)+(I-J) e(k h)] \\
& \left.+\|(I-J) G\|^{2}\right] d t \\
& -\left(W^{T}(t)-W^{T}(k h)\right) G(I-J) \\
& \times L[\delta(k h)+(I-J) e(k h)] \\
& +\delta^{T}(t)(I-J) G d W(t) \\
\leq & \left(h \lambda_{n}[\delta(k h)+(I-J) e(k h)]^{T}\right. \\
& \times L[\delta(k h)+(I-J) e(k h)] \\
& +\delta^{T}(t)(I-J) G d W(t) \\
& \left.+\|(I-J) G\|^{2}\right) d t \\
& \left(\delta^{T}(t)-W^{T}(k h)\right) G(I-J) \\
& L[\delta(k h)+(I-J) e(k h)] \\
& +k h)+(I-J) e(k h)] \\
& \left.+{ }^{T}\right) \\
&
\end{aligned}
$$




$$
\begin{aligned}
= & \left(\left(h \lambda_{n}-1\right) \delta^{T}(k h) L \delta(k h)+\left(2 h \lambda_{n}-1\right)\right. \\
& \times \delta^{T}(k h) L(I-J) e(k h) \\
& +h \lambda_{n}\|I-J\|^{2} e^{T}(k h) L e(k h) \\
& \left.+\|(I-J) G\|^{2}\right) d t \\
& -\left(W^{T}(t)-W^{T}(k h)\right) G(I-J) \\
& \times L[\delta(k h)+(I-J) e(k h)] \\
& +\delta^{T}(t)(I-J) G d W(t)
\end{aligned}
$$

Applying the inequality

$$
\begin{aligned}
\delta^{T}(k h) L(I-J) e(k h) \leq & \frac{1}{2}\|L\|^{2} \delta^{T}(k h) \delta(k h) \\
& +\frac{1}{2}\|I-J\|^{2} e^{T}(k h) e(k h),
\end{aligned}
$$

it can be derived that

$$
\begin{aligned}
d V(\delta(t), t) \leq & \left(-\frac{1}{2} \delta^{T}(k h) L \delta(k h)\right. \\
& +\frac{1}{2}\|I-J\|^{2} e^{T}(k h) L e(k h) \\
& \left.+\|(I-J) G\|^{2}\right) d t \\
& -\left(W^{T}(t)-W^{T}(k h)\right) G(I-J) \\
& \times L[\delta(k h)+(I-J) e(k h)] \\
& +\delta^{T}(t)(I-J) G d W(t)
\end{aligned}
$$

with $2 h \lambda_{n} \leq 1$.

We can see that

$$
\begin{aligned}
d\left[e^{-t} V(\delta(t), t)\right] & \\
= & e^{-t}[-V(\delta(t), t) d t+d V(\delta(t), t)] \\
\leq & e^{-t}\left[-V(\delta(t), t)-\frac{1}{2} \delta^{T}(k h) L \delta(k h)\right. \\
& +\frac{1}{2}\|I-J\|^{2} e^{T}(k h) L e(k h) \\
& \left.+\|(I-J) G\|^{2}\right] d t \\
- & e^{-t}\left(W^{T}(t)-W^{T}(k h)\right) G(I-J) \\
& \times L[\delta(k h)+(I-J) e(k h)] \\
& +e^{-t} \delta^{T}(t)(I-J) G d W(t) .
\end{aligned}
$$

Integrating both sides of the previous inequality from $k h$ to $t(t \in[k h,(k+1) h))$, making use of $(9)$, and taking the expectation, one can obtain that

$$
\begin{aligned}
e^{-t} \mathbb{E} V(\delta(t), t) \leq & \int_{k h}^{t}-e^{-t} \mathbb{E} V(\delta(t), t) d t \\
& +\frac{1}{2} e^{-k h} \delta^{T}(k h) \delta(k h) \\
& -\left(\frac{1}{2} \delta^{T}(k h) L \delta(k h)\right. \\
& -\frac{1}{2}\|I-J\|^{2} e^{T}(k h) L e(k h) \\
& \left.-\|(I-J) G\|^{2}\right)\left(e^{-t}-e^{-k h}\right) \\
\leq & \int_{k h}^{t}-e^{-t} \mathbb{E} V(\delta(t), t) d t \\
& +\frac{1}{2} e^{-k h} \delta^{T}(k h) \delta(k h) \\
& +\left(\frac{1}{2} \delta^{T}(k h) L \delta(k h)\right. \\
& -\frac{1}{2}\|I-J\|^{2} e^{T}(k h) L e(k h) \\
& +\int_{k h}^{t}-e^{-t} \mathbb{E} V(\delta(t), t) d t \\
& \left.-\|(I-J) G\|^{2}\right)\left(e^{-k h}-e^{-(k+1) h}\right) \\
= & \int_{k h}^{t}-e^{-t} \mathbb{E} V(\delta(t), t) d t \\
& +\frac{1}{2} e^{-k h} \delta^{T}(k h) \delta(k h) \delta(k h) . \\
& +\left(\frac{1}{2}\|I-J\|^{2} x^{T}(k h) L x(k h)\right. \\
& -\|I-J\|^{2} e^{T}(k h) L e(k h)
\end{aligned}
$$

By using Gronwall inequality, we have

$$
\mathbb{E} V(\delta(t), t) \leq \frac{1}{2} e^{-k h}\|\delta(k h)\|^{2} e^{-(t-k h)}=\frac{1}{2}\|\delta(k h)\|^{2} e^{-t} .
$$

Letting $t \rightarrow \infty(k \rightarrow \infty)$, we obtain

$$
\lim _{t \rightarrow \infty} \mathbb{E}\|\delta(t)\|^{2}=0
$$

So the system (8) reaches average consensus in mean square. 


\subsection{WSNs with Markovian Switching Topology}

Theorem 3. Consider the system (16) over a number of connected switching communication graphs driven by event condition in (18). Then the system (17) is asymptotically stable in mean square; it means that the system (16) reaches average consensus in mean square if $0 \leq h \leq 1 / 2 \lambda_{n}(r(t))$.

Proof. Define the function $V: R^{n} \times R_{+} \times S \rightarrow R^{+}$by

$$
V(\delta, t, r(t))=\frac{1}{2} \delta^{T}(t) \delta(t) .
$$

Computing $d V(\delta, t, r(t))$ along the trajectory generated by the system (11) for any $t \in[k h,(k+1) h)$, we have

$$
\begin{aligned}
& d V(\delta, t, r(t))=\left[\delta^{T}(t) f(\delta, t, r(t))\right. \\
& \left.+\operatorname{trace}\left[g^{T}(\delta, t, r(t)) g(\delta, t, r(t))\right]\right] d t \\
& +\delta^{T}(t)(I-J) G(r(t)) d W(t) \\
& =\left[-\delta^{T}(t) L(r(t))[\delta(k h)+(I-J) e(k h)]\right. \\
& \left.+\|(I-J) G\|^{2}+\sum_{j=1}^{N} \gamma_{i j} V(\delta, t, r(t))\right] d t \\
& +\delta^{T}(t)(I-J) G(r(t)) d W(t) \\
& \leq\left(\left(h \lambda_{n}-1\right) \delta^{T}(k h) L(r(t)) \delta(k h)\right. \\
& +\left(2 h \lambda_{n}-1\right) \delta^{T}(k h) L(r(t))(I-J) e(k h) \\
& +h \lambda_{n}\|I-J\|^{2} e^{T}(k h) L(r(t)) e(k h) \\
& \left.+\|(I-J) G(r(t))\|^{2}+\bar{\gamma} V(\delta, t, r(t))\right) d t \\
& -\left(W^{T}(t)-W^{T}(k h)\right) G(r(t))(I-J) \\
& \times L(r(t))[\delta(k h)+(I-J) e(k h)] \\
& +\delta^{T}(t)(I-J) G(r(t)) d W(t) \\
& \leq\left(-\frac{1}{2} \delta^{T}(k h) L(r(t)) \delta(k h)\right. \\
& +\frac{1}{2}\|I-J\|^{2} e^{T}(k h) L(r(t)) e(k h) \\
& \left.+\|(I-J) G(r(t))\|^{2}+\bar{\gamma} V(\delta, t, r(t))\right) d t \\
& -\left(W^{T}(t)-W^{T}(k h)\right) G(r(t))(I-J) \\
& \times L(r(t))[\delta(k h)+(I-J) e(k h)] \\
& +\delta^{T}(t)(I-J) G(r(t)) d W(t)
\end{aligned}
$$

For $\bar{\gamma}>0, \bar{\gamma}=\max \left\{\left|\gamma_{i i}: i \in S\right|\right\}$, compute

$$
\begin{gathered}
d\left[e^{-2 \bar{\gamma} t} V(\delta(t), t, r(t))\right] \\
=e^{-2 \bar{\gamma} t}[-2 \bar{\gamma} V(\delta(t), t, r(t)) d t+d V(\delta(t), t, r(t))] \\
\leq e^{-2 \bar{\gamma} t}[-2 \bar{\gamma} V(\delta(t), t, r(t)) \\
\quad-\frac{1}{2} \delta^{T}(k h) L(r(t)) \delta(k h) \\
\quad+\frac{1}{2}\|I-J\|^{2} e^{T}(k h) L(r(t)) e(k h) \\
\left.\quad+\|(I-J) G(r(t))\|^{2}+\bar{\gamma} V(\delta, t, r(t))\right] d t \\
-e^{-2 \bar{\gamma} t}\left(W^{T}(t)-W^{T}(k h)\right) G(I-J) \\
\quad \times L(r(t))[\delta(k h)+(I-J) e(k h)] \\
+e^{-2 \bar{\gamma} t} \delta^{T}(t)(I-J) G(r(t)) d W(t) .
\end{gathered}
$$

Integrating both sides of the above inequality from $k h$ to $t(t \in[k h,(k+1) h))$, making use of (18), and taking the expectation, one can obtain that

$$
\begin{aligned}
& e^{-2 \bar{\gamma} t} \mathbb{E} V(\delta(t), t, r(t)) \\
& \leq \int_{k h}^{t}-\bar{\gamma} e^{-2 \bar{\gamma} t} \mathbb{E} V(\delta(t), t, r(t)) d t \\
& +\frac{1}{2} e^{-2 \bar{\gamma} k h} \delta^{T}(k h) \delta(k h) \\
& -\left(\frac{1}{2} \delta^{T}(k h) L(r(t)) \delta(k h)\right. \\
& -\frac{1}{2}\|I-J\|^{2} e^{T}(k h) L(r(t)) e(k h) \\
& \left.-\|(I-J) G(r(t))\|^{2}\right)\left(e^{-2 \bar{\gamma} t}-e^{-2 \bar{\gamma} k h}\right) \\
& \leq \int_{k h}^{t}-\bar{\gamma} e^{-2 \bar{\gamma} t} \mathbb{E} V(\delta(t), t, r(t)) d t \\
& +\frac{1}{2} e^{-2 \bar{\gamma} k h} \delta^{T}(k h) \delta(k h) \\
& +\left(\frac{1}{2} \delta^{T}(k h) L(r(t)) \delta(k h)\right. \\
& -\frac{1}{2}\|I-J\|^{2} e^{T}(k h) L(r(t)) e(k h) \\
& \left.-\|(I-J) G(r(t))\|^{2}\right) \\
& \times\left(e^{-2 \bar{\gamma} k h}-e^{-2 \bar{\gamma}(k+1) h}\right)
\end{aligned}
$$

with $2 h \lambda_{n} \leq 1$. 


$$
\begin{aligned}
& =\int_{k h}^{t}-\bar{\gamma} e^{-2 \bar{\gamma} t} \mathbb{E} V(\delta(t), t, r(t)) d t \\
& +\frac{1}{2} e^{-2 \bar{\gamma} k h} \delta^{T}(k h) \delta(k h) \\
& +\left(\frac{1}{2}\|I-J\|^{2} x^{T}(k h) L(r(t)) x(k h)\right. \\
& \quad-\frac{1}{2}\|I-J\|^{2} e^{T}(k h) L(r(t)) \\
& \left.\quad \times e(k h)-\|(I-J) G(r(t))\|^{2}\right) \\
& \quad \times\left(e^{-2 \bar{\gamma} k h}-e^{-2 \bar{\gamma}(k+1) h)}\right. \\
& \leq \int_{k h}^{t}-\bar{\gamma} e^{-2 \bar{\gamma} t} \mathbb{E} V(\delta(t), t, r(t)) d t \\
& +\frac{1}{2} e^{-2 \bar{\gamma} k h} \delta^{T}(k h) \delta(k h) .
\end{aligned}
$$

By using Gronwall inequality, we have

$$
\begin{aligned}
\mathbb{E} V(\delta(t), t, r(t)) & \leq \frac{1}{2} e^{-2 \bar{\gamma} k h}\|\delta(k h)\|^{2} e^{-\bar{\gamma}(t-k h)} \\
& =\frac{1}{2} e^{-\bar{\gamma}(t+k h)}\|\delta(k h)\|^{2}
\end{aligned}
$$

Letting $t \rightarrow \infty(k \rightarrow \infty)$, we obtain that

$$
\lim _{t \rightarrow \infty} \mathbb{E}\|\delta(t)\|^{2}=0
$$

So the system (16) reaches average consensus in mean square.

\section{Numerical Examples}

In this section, we give two examples to examine the average consensus of the systems (8) and (16).

Example 1. Consider a WSN composed by four sensors in which each dynamic state of the sensor is $\dot{x}_{i}=u_{i}$, where $i=1,2,3,4$ (see the topology in Figure 1, which is used in Dimarogonas [16]) and the initial state is $X(0)=(4-1,5,2)^{T}$.

The adjacent matrix is

$$
A=\left(\begin{array}{llll}
0 & 1 & 1 & 0 \\
1 & 0 & 1 & 0 \\
1 & 1 & 0 & 1 \\
0 & 0 & 1 & 0
\end{array}\right)
$$

The related degree matrix is

$$
D=\left(\begin{array}{llll}
2 & 0 & 0 & 0 \\
0 & 2 & 0 & 0 \\
0 & 0 & 3 & 0 \\
0 & 0 & 0 & 1
\end{array}\right)
$$

The related Laplacian matrix is

$$
L=\left(\begin{array}{cccc}
2 & -1 & -1 & 0 \\
-1 & 2 & -1 & 0 \\
-1 & -1 & 3 & -1 \\
0 & 0 & -1 & 1
\end{array}\right)
$$

The nonzero largest and smallest eigenvalues are $\lambda_{n}=4$ and $\lambda_{2}=1$, respectively.

Let the noise intensity matrix $G$ be

$$
G=\left(\begin{array}{cccc}
0.001 & 0 & 0 & 0 \\
0 & 0.002 & 0 & 0 \\
0 & 0 & 0.003 & 0 \\
0 & 0 & 0 & 0.001
\end{array}\right)
$$

The sampling period for all sensor nodes is chosen as $h=0.003$ which satisfies $0 \leq h \leq 1 / 2 \lambda_{n}$. Using the event condition in (9), we can draw the dynamic curve of the states of the sensors by Matlab as Figure 2. It shows us that the four sensor nodes reach the average consensus in mean square with fixed topology. The control signal when the events occur for each sensor is shown in Figure 3. We can see that the number of sensor control updates that only occur at the sampling instant under the event trigger condition is rapidly reduced to reach average consensus.

Example 2. The switching topology of the four sensors is determined by the Markov chain $r(t)$ whose state space is $S=\{1,2,3\}$. The related topology graph is $\mathscr{G}(i)=$ $\{\mathscr{V}(i), \mathscr{E}(i), \mathscr{A}(i)\}$ (see the topologies in Figure 4).

The adjacent matrices are

$$
\begin{gathered}
A(1)=\left(\begin{array}{llll}
0 & 1 & 1 & 0 \\
1 & 0 & 0 & 0 \\
1 & 0 & 0 & 1 \\
0 & 0 & 1 & 0
\end{array}\right), \quad A(2)=\left(\begin{array}{llll}
0 & 1 & 0 & 0 \\
1 & 0 & 0 & 1 \\
0 & 0 & 0 & 1 \\
0 & 1 & 1 & 0
\end{array}\right), \\
A(3)=\left(\begin{array}{llll}
0 & 0 & 1 & 0 \\
0 & 0 & 0 & 1 \\
1 & 0 & 0 & 1 \\
0 & 1 & 1 & 0
\end{array}\right) .
\end{gathered}
$$

The related degree matrices are

$$
\begin{gathered}
D(1)=\left(\begin{array}{llll}
2 & 0 & 0 & 0 \\
0 & 1 & 0 & 0 \\
0 & 0 & 2 & 0 \\
0 & 0 & 0 & 1
\end{array}\right), \quad D(2)=\left(\begin{array}{llll}
1 & 0 & 0 & 0 \\
0 & 2 & 0 & 0 \\
0 & 0 & 1 & 0 \\
0 & 0 & 0 & 2
\end{array}\right), \\
D(3)=\left(\begin{array}{llll}
1 & 0 & 0 & 0 \\
0 & 1 & 0 & 0 \\
0 & 0 & 2 & 0 \\
0 & 0 & 0 & 2
\end{array}\right) .
\end{gathered}
$$




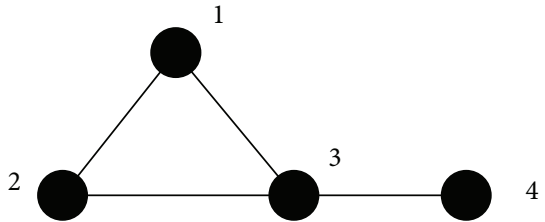

Figure 1: The topology of the four sensors.

The related Laplacian matrices are

$$
\begin{aligned}
& L(1)=\left(\begin{array}{cccc}
2 & -1 & -1 & 0 \\
-1 & 1 & 0 & 0 \\
-1 & 0 & 2 & -1 \\
0 & 0 & -1 & 1
\end{array}\right), \\
& L(2)=\left(\begin{array}{cccc}
1 & -1 & 0 & 0 \\
-1 & 2 & 0 & -1 \\
0 & 0 & 1 & -1 \\
0 & -1 & -1 & 2
\end{array}\right), \\
& L(3)=\left(\begin{array}{cccc}
1 & 0 & -1 & 0 \\
0 & 1 & 0 & -1 \\
-1 & 0 & 2 & -1 \\
0 & -1 & -1 & 2
\end{array}\right) .
\end{aligned}
$$

The nonzero largest and smallest eigenvalue are $\lambda_{n}=$ 3.4142 and $\lambda_{2}=0.5858$, respectively.

Let the noise intensity matrices $G$ be

$$
\begin{aligned}
& G(1)=\left(\begin{array}{cccc}
0.001 & 0 & 0 & 0 \\
0 & 0.002 & 0 & 0 \\
0 & 0 & 0.003 & 0 \\
0 & 0 & 0 & 0.001
\end{array}\right), \\
& G(2)=\left(\begin{array}{cccc}
0.002 & 0 & 0 & 0 \\
0 & 0.003 & 0 & 0 \\
0 & 0 & 0.003 & 0 \\
0 & 0 & 0 & 0.001
\end{array}\right), \\
& G(3)=\left(\begin{array}{cccc}
0.003 & 0 & 0 & 0 \\
0 & 0.002 & 0 & 0 \\
0 & 0 & 0.001 & 0 \\
0 & 0 & 0 & 0.001
\end{array}\right) .
\end{aligned}
$$

The sampling period for all sensor nodes is chosen as $h=$ 0.002 which satisfies $0 \leq h \leq 1 / 2 \lambda_{n}(i)(i=1,2,3)$. Using the event condition in (18), we can draw the dynamic curve of the states of the sensors by Matlab as Figure 5. It shows us that the four sensor nodes reach the average consensus in mean square with Markovian switching topologies.

\section{Conclusions}

In this paper, we have dealt with the problem of average consensus in mean square of the WSNs. By using the eventbased control mechanism, we have obtained several sufficient conditions to ensure the average consensus in mean square for WSNs with fixed and Markovian switching topologies. There are many other topics worth investigating, such as the

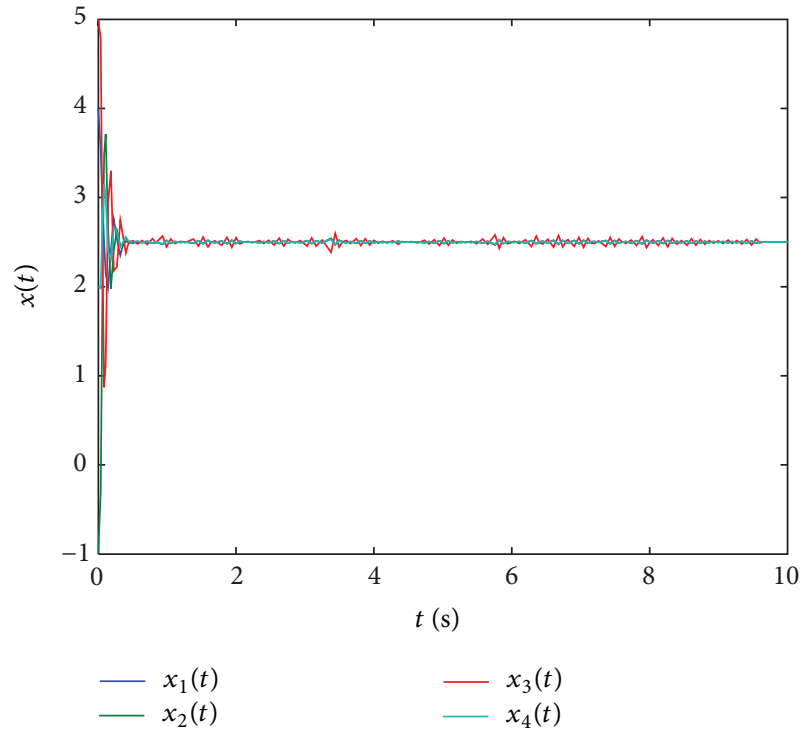

Figure 2: The trajectories of the state vectors $x_{1}(t), x_{2}(t), x_{3}(t)$, and $x_{4}(t)$.

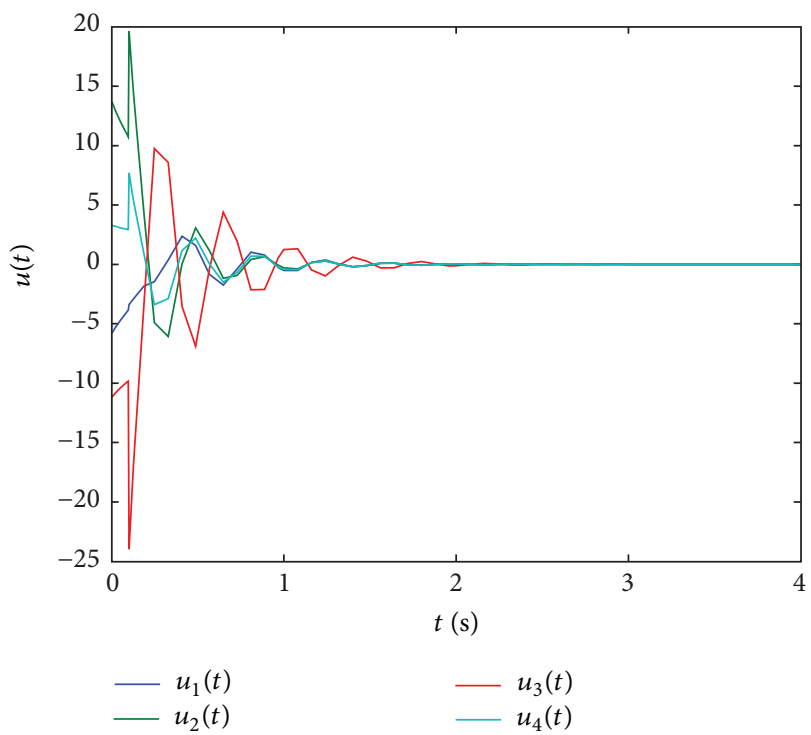

FIGURE 3: Control inputs for the sensors.

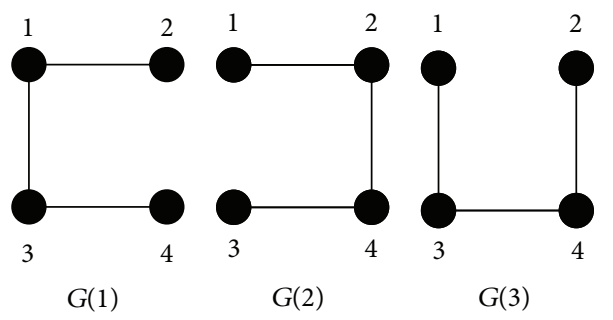

(a)

(b)

(c)

FIGURE 4: The topologies of the four sensors in states 1, 2, and 3. 


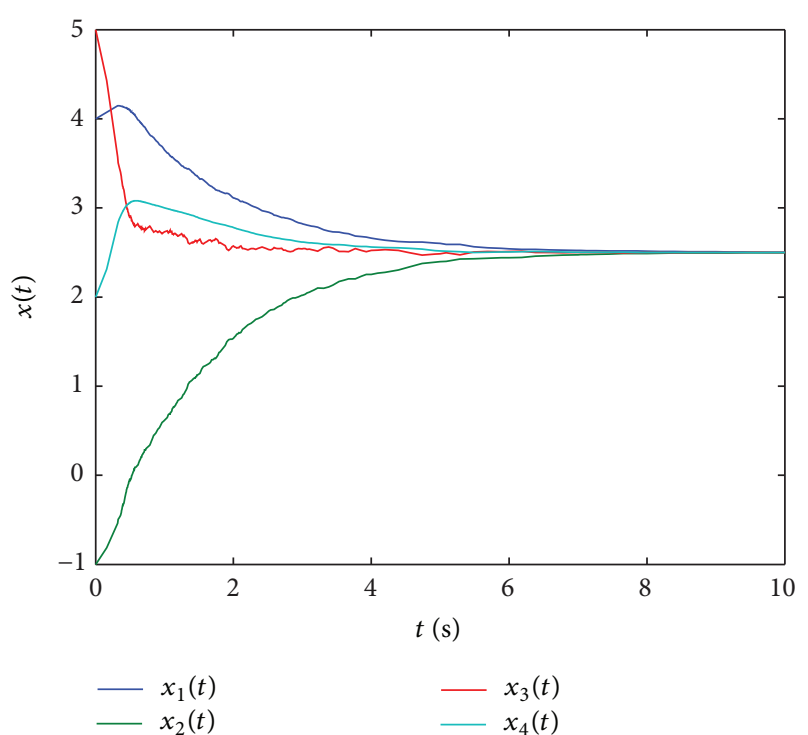

Figure 5: The trajectories of the state vectors $x_{1}(t), x_{2}(t), x_{3}(t)$, and $x_{4}(t)$.

packet loss and time-delay cases of the WSN's consensus problem under event condition.

\section{Acknowledgments}

This work is supported by the National Natural Science Foundation of China $(61075060,61203337)$, the Innovation Program of Shanghai Municipal Education Commission (12zz064), the Specialized Research Fund for the Doctoral Program of Higher Education under Grant no. 20120075120009, and the Natural Science Foundation of Shanghai (12ZR1440200).

\section{References}

[1] J. A. Fax and R. M. Murray, "Information flow and cooperative control of vehicle formations," IEEE Transactions on Automatic Control, vol. 49, no. 9, pp. 1465-1476, 2004.

[2] R. Olfati-Saber, "Distributed Kalman filter with embedded consensus filters," in Proceedings of the 44th IEEE Conference on Decision and Control, and the European Control Conference (CDC-ECC '05), pp. 8179-8184, Seville, Spain, December 2005.

[3] N. Lynch, Distributed Algorithms, Morgan Kaufmann, San Matero, Calif, USA, 1996.

[4] R. Olfati-Saber and R. M. Murray, "Consensus problems in networks of agents with switching topology and time-delays," IEEE Transactions on Automatic Control, vol. 49, no. 9, pp. 15201533, 2004.

[5] D. B. Kingston and R. W. Beard, "Discrete-time averageconsensus under switching network topologies," in Proceedings of the American Control Conference, pp. 3551-3556, Minneapolis, Minn, USA, June 2006.

[6] L. Xiao and S. Boyd, "Fast linear iterations for distributed averaging," Systems and Control Letters, vol. 53, no. 1, pp. 65-78, 2004.
[7] Q. Zhang and J. Zhang, "Distributed consensus of continuoustime multi-agent systems with markovian switching topologies and stochastic communication noises," Journal of Systems Science and Mathematical Sciences, vol. 31, no. 9, pp. 1097-1110, 2011.

[8] Z. Wang, D. Ding, H. Dong, and H. Shu, "Ho consensus control for multi-agent systems with missing measurements: the finite-horizon case," Systems and Control Letters, vol. 62, no. 10, pp. 827-836, 2013.

[9] D. Ding, Z. Wang, H. Dong, and H. Shu, "Distributed Ho state estimation with stochastic parameters and nonlinearities through sensor networks: the finite-horizon case," Automatica, vol. 48, no. 8, pp. 1575-1585, 2012.

[10] H. Dong, Z. Wang, and H. Gao, "Distributed Ho filtering for a class of Markovian jump nonlinear time-delay systems over lossy sensor networks," IEEE Transactions on Industrial Electronics, vol. 60, no. 10, pp. 4665-4672, 2013.

[11] H. Dong, Z. Wang, and H. Gao, "Distributed filtering for a class of time-varying systems over sensor networks with quantization errors and successive packet dropouts," IEEE Transactions on Signal Processing, vol. 60, no. 6, pp. 3164-3173, 2012.

[12] X. Wang and M. D. Lemmon, "Event-triggering in distributed networked control systems," IEEE Transactions on Automatic Control, vol. 56, no. 3, pp. 586-601, 2011.

[13] M. Mazo and P. Tabuada, "Decentralized event-triggered control over wireless sensor/actuator networks," IEEE Transactions on Automatic Control, vol. 56, no. 10, pp. 586-601, 2011.

[14] G. Seyboth, D. Dimarogonas, and K. Johansson, "Event-based broadcasting for multi-agent average consensus," Automatica, vol. 49, no. 1, pp. 245-252, 2013.

[15] X. Meng and T. Chen, "Event based agreement protocols for multi-agent networks," Automatica, vol. 49, no. 7, pp. 2125-2132, 2013.

[16] D. V. Dimarogonas, E. Frazzoli, and K. H. Johansson, "Distributed event-triggered control for multi-agent systems," IEEE Transactions on Automatic Control, vol. 57, no. 5, pp. 1291-1297, 2012. 


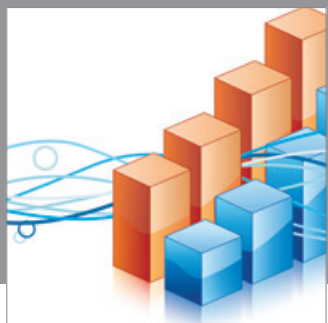

Advances in

Operations Research

mansans

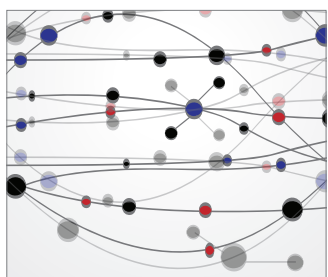

The Scientific World Journal
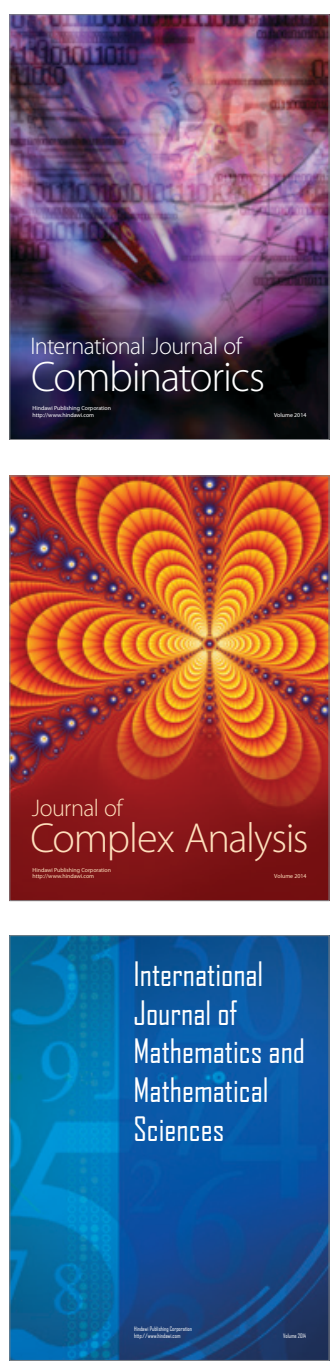
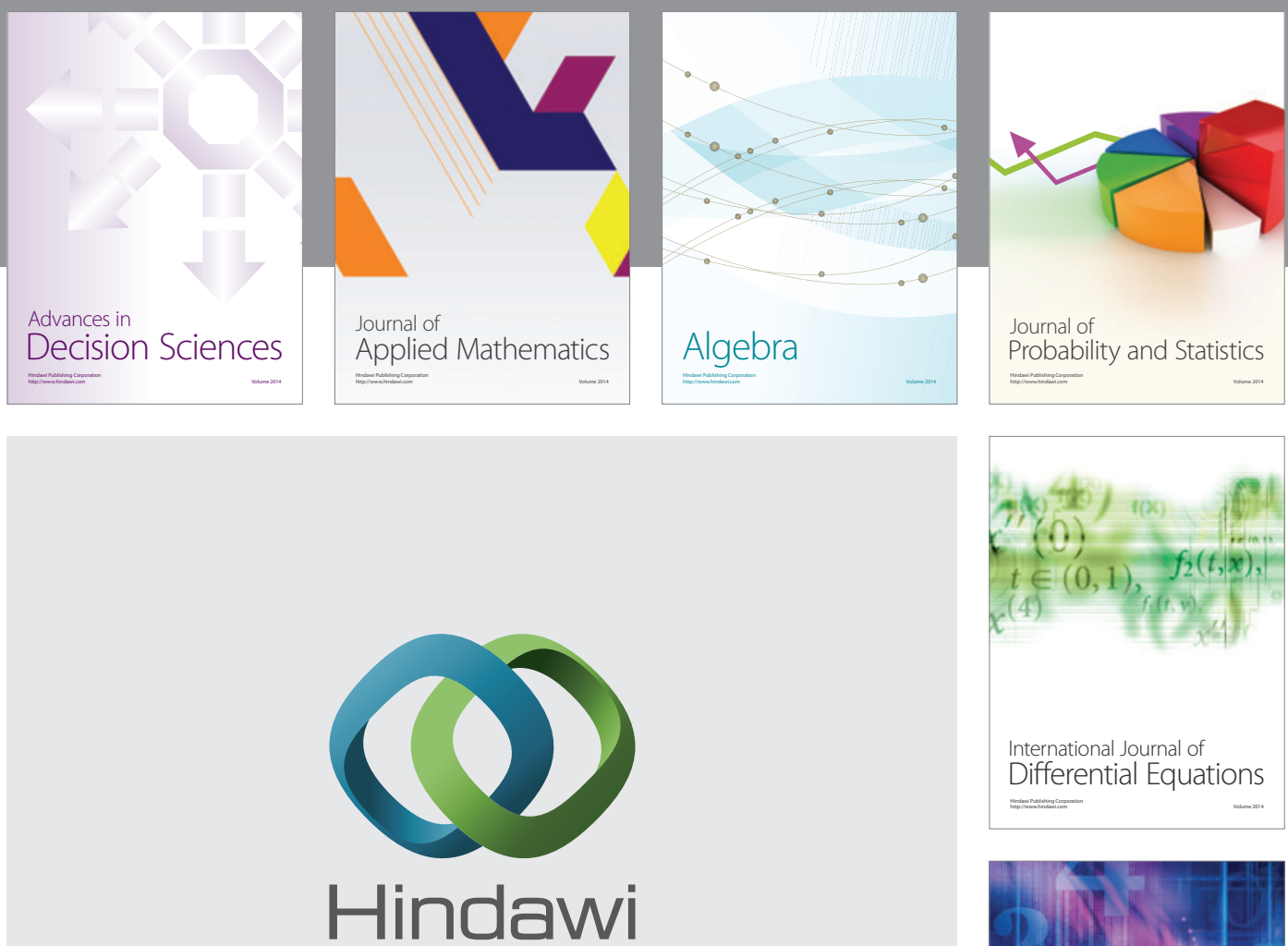

Submit your manuscripts at http://www.hindawi.com
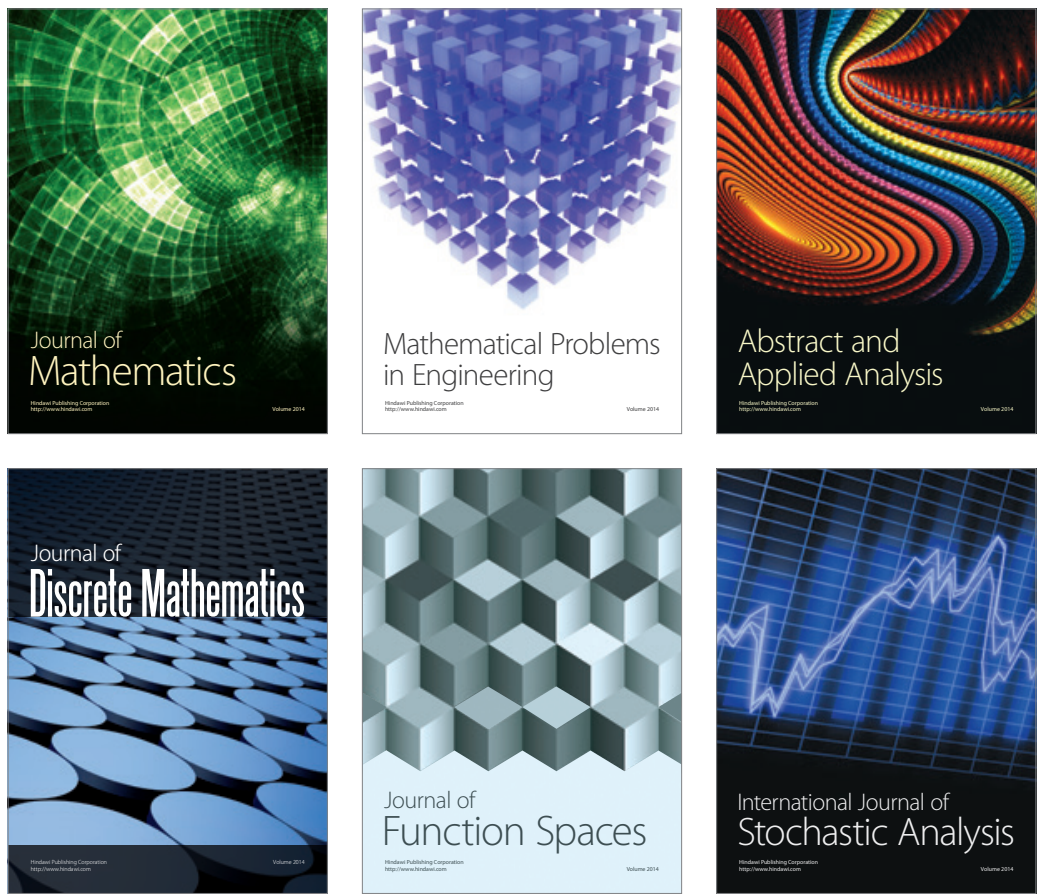

Journal of

Function Spaces

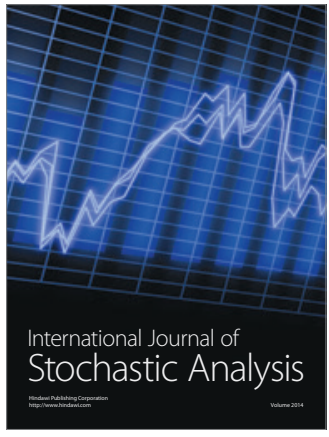

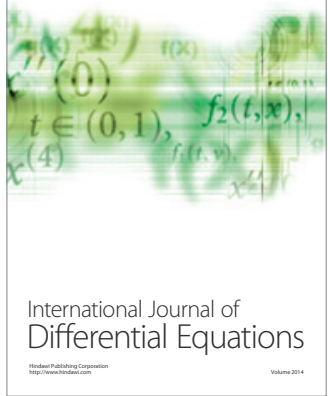
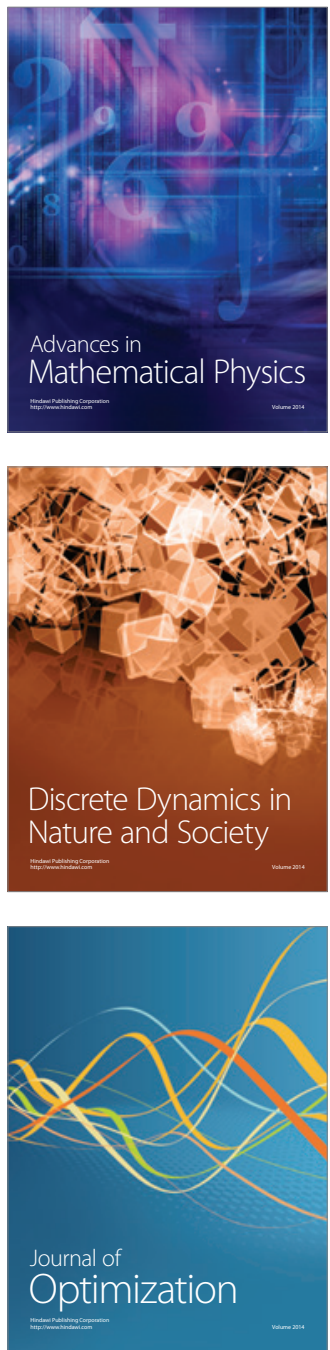